\title{
Pharmacokinetic optimisation of $\beta$-lactams for the treatment of ventilator-associated pneumonia
}

\author{
G.L. Drusano
}

ABSTRACT: Rational therapy of ventilator-associated pneumonia requires choosing the right drug at the right dose. The choice of dose depends on the microbiological goal, the range of minimum inhibitory concentrations (MICs) for likely pathogens, the extent of the drug's protein binding and, in humans, pharmacokinetics.

If protein binding and the distribution of pharmacokinetic parameters are known, as well as the likely pathogens and MICs in a given setting, Monte Carlo simulations can be used to calculate the likelihood that a given dose will attain an identified microbiological goal. For $\beta$-lactams, the antibacterial effect depends on the percentage of time (\%T) during the dosing interval that the free (nonprotein bound) antibiotic concentration remains above the MIC (\%T >MIC); the required values are smaller for carbapenems than for penicillins or cephalosporins.

Data show that infusion of an i.v. $\beta$-lactam over an extended period may greatly increase \%T $>$ MIC, and hence efficacy, at a given dose. Alternatively, it may provide the same efficacy at a lower total dose, reducing cost and potential toxicity. In a real-life setting, a 4-h infusion of $3.375 \mathrm{~g}$ piperacillin-tazobactam $98 \mathrm{~h}$ (every $8 \mathrm{~h}$; 3 times daily); proved more effective for severe Pseudomonas aeruginosa infections than a 30-min infusion of $3.375 \mathrm{~g} \mathrm{q6} \mathrm{h}$ (every $6 \mathrm{~h}$; 4 times daily) or $q 4 h$ (every $4 \mathrm{~h} ; 6$ times daily).

Data for doripenem, an investigational carbapenem, suggest that its stability following reconstitution may render it particularly suitable for extended infusion.

KEYWORDS: Appropriate antibiotic therapy, $\beta$-lactams, broad-spectrum antibiotics, nosocomial pneumonia, ventilator-associated pneumonia

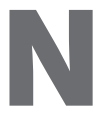

osocomial pneumonia, including the $86 \%$ of cases associated with ventilator use [1], is the leading cause of death among hospital infections, with reported mortality rates ranging $24-76 \%$ [2]. Stays in intensive care units (ICUs) are extended for those who survive $[1,3]$. According to guidelines from the American Thoracic Society and the Infectious Diseases Society of America, treatment with a broadspectrum antibiotic should be initiated as soon as nosocomial pneumonia is suspected, followed by a switch to more targeted therapy once the causative organism and its susceptibilities have been identified [4].

The first step in the treatment of ventilatorassociated pneumonia (VAP) is choosing the correct dose of the correct drug. Once the drug is chosen, the rational choice of the dose involves four considerations: 1) the microbiological goal of therapy (e.g. 1-log kill, 2-log kill); 2) the minimum inhibitory concentration (MIC) distribution of the target pathogen or pathogens; 3 ) the extent to which the administered drug becomes bound to body proteins; and 4) the drug's pharmacokinetics in the target population.

The microbiological goal of therapy is not automatic: sometimes it is enough to halt bacterial growth, allowing the body's immune system to eradicate the pathogen. In other cases, it is necessary to kill $90-99 \%$ of the pathogens in order for the immune system to do its job properly. It is important to determine which goal is appropriate for a given situation and choose the dose accordingly.

Although the choice of dose depends on the MIC of the pathogen, the precise pathogen and the drug's MIC is usually not known at the time of initial, empiric therapy. The physician must consider a range of possibilities. The range will not be the same for every institution and perhaps not even for every setting within the same institution. It is important to know which pathogens the

\section{AFFILIATIONS}

Ordway Research Institute, Albany, NY, USA.

CORRESPONDENCE

G.L. Drusano

Ordway Research Institute

150 New Scotland Avenue

Albany

New York 12208

NY

USA

Fax: 15186416304

E-mail: gdrusano@

ordwayresearch.org

STATEMENT OF INTEREST

G.L. Drusano has received

educational and research funds and has served as a consultant for Johnson \& Johnson Pharmaceutical Research and Development. 


\begin{tabular}{|c|c|c|}
\hline TABLE 1 & \multicolumn{2}{|c|}{$\begin{array}{l}\text { Percentage of tıme durıng the dosing interval spent above the mınımal Innibitory concentration, required tor } \beta \text {-lactam } \\
\text { antibiotic efficacy }\end{array}$} \\
\hline Drug class & Stasis target (prevents pathogen growth) & 2-Log kill target (near-maximal kill) \\
\hline Carbapenems & 20 & 40 \\
\hline Penicillins & 30 & 50 \\
\hline Cephalosporins & 40 & $60-70$ \\
\hline
\end{tabular}

Reproduced from [5] with permission from the publisher.

patient is likely to have been exposed to and the range of MICs that those pathogens are likely to exhibit. A dose must then be chosen that will cover all likely pathogens and susceptibilities without high drug toxicities and costs.

It is also important to know how much of the administered drug binds to plasma proteins and other body proteins. In general, only unbound drug is free to act on the pathogen and to be metabolised and excreted.

The pharmacokinetics of a drug in an individual patient are often overlooked, yet they may vary from patient to patient. Besides kidney and liver function, age, sex, body size, fat/ muscle ratio and the genetic variability of metabolic enzymes can affect pharmacokinetics. Although such factors may be difficult for the physician to evaluate in each case, they should be considered when deciding on the appropriate treatment dose.

\section{ANTIMICROBIAL PHARMACODYNAMICS: EXPOSURE TARGET}

For $\beta$-lactam antibiotics, the ability to stop the growth of an organism, or to kill it, depends on the percentage of time (\%T) during the dosing interval that the free drug concentration remains above the MIC (\%T $>$ MIC). Table 1 is based on extensive research in a mouse thigh infection model (personal communication; W.A. Craig, University of Wisconsin, Madison, WI, USA) and illustrates how the different classes

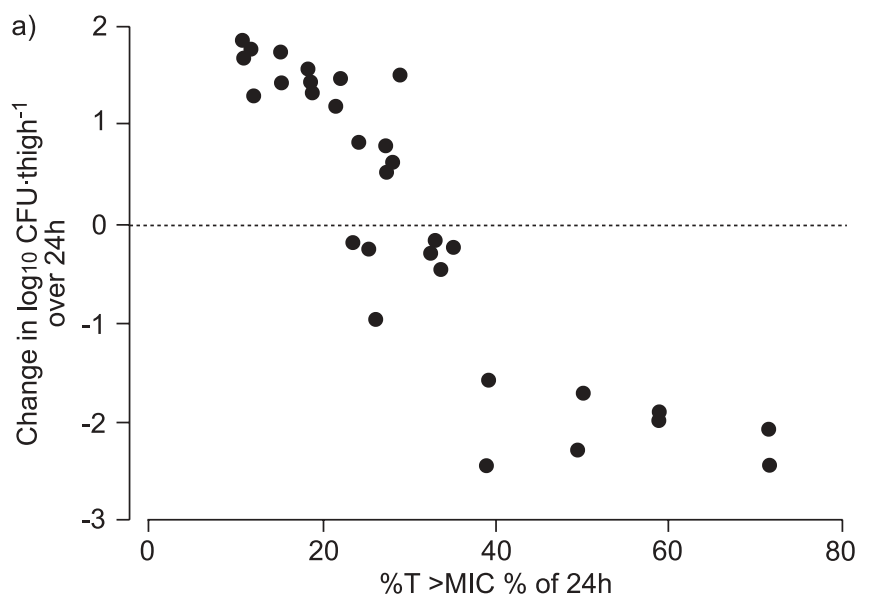

of $\beta$-lactams compare. For a wide variety of dosing intervals, the required $\% \mathrm{~T}>\mathrm{MIC}$ for bacteriostasis is $\sim 20 \%$ for carbapenems, 30\% for penicillins and $40 \%$ for cephalosporins. The $\% \mathrm{~T}>$ MIC for maximal killing is $\sim 20 \%$ greater for each antibiotic [5]. For example, as shown in figure 1, meropenem produced bacteriostasis at $\sim 20 \% \mathrm{~T}>\mathrm{MIC}$ (when the log of the change in colony forming units (CFU) thigh $^{-1}$ was zero) and a 2-log killing effect at $\sim 40 \% \mathrm{~T}>\mathrm{MIC}$. This was true regardless of whether the drug was tested against Escherichia coli or Pseudomonas aeruginosa. Although the MIC and actual dose were different for E. coli and P. aeruginosa, the \% $>\mathrm{TIC}$ required to achieve the same microbiological goals was the same [5].

\section{PHARMACODYNAMICS: PREDICTION OF MICROBIOLOGICAL EFFICACY}

If the goal of antibiotic therapy is to eliminate all but a remnant of the pathogen, a remnant that most patients' immune systems would be able to remove without difficulty, the concentration of a carbapenem antibiotic would have to be $>$ MIC for $\geqslant 40 \%$ of the dosing interval. What this means in terms of actual dose depends both on the pathogen's MIC and on the pharmacokinetics of the drug in the individual patient. The goal will be harder to achieve if the MIC of the pathogen is 4 or $8 \mathrm{mg} \cdot \mathrm{L}^{-1}$ rather than $0.25 \mathrm{mg} \cdot \mathrm{L}^{-1}$, and in a young person with good kidney function (and more rapid drug clearance) than in an older adult with reduced kidney function.

b)

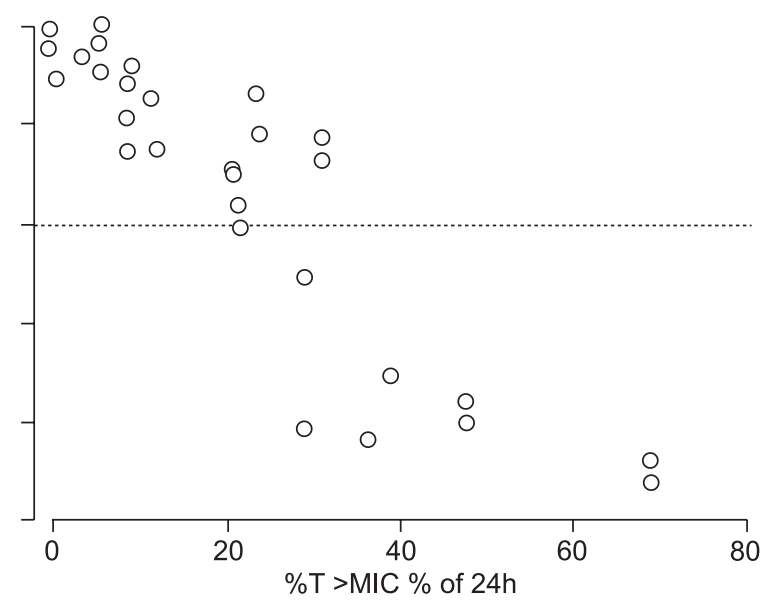

FIGURE 1. Relationship between the percentage of time (\%T) spent above the minimum inhibitory concentration (MIC) and efficacy in an experimental mouse infection model. The logarithm of the change in the number of colony forming units (CFU) of pathogen in an infected thigh (Escherichia coli $(\bullet)$ or Pseudomonas aeruginosa $(\bigcirc)$ ) is shown as a function of the \%T that the plasma level of meropenem was $>$ MIC. .....: bacteriostasis, no net change in the number of CFUs. Points below this line approximate to a $99 \%$ (2-log unit) kill rate. Reproduced from [5] with permission from the publisher. 


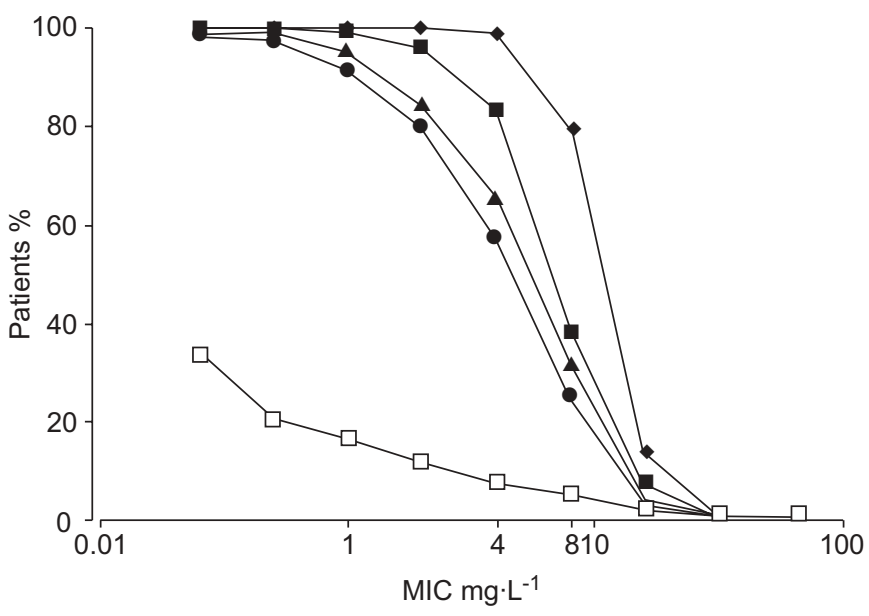

FIGURE 2. Percentage of patients attaining the target of $40 \%$ of dosage time above the mean inhibitory concentration (MIC), treated with 1,000 mg meropenem to maximise kill and infusion durations of $0.5(\bullet), 1.0(\mathbf{\bullet}), 2.0(\mathbf{\square})$ and $3.0 \mathrm{~h}(\bullet)$. $\square$ percentage of Pseudomonas aeruginosa isolates with the respective MIC, the greatest percentage is at low MICs and the smallest percentage at high MICs. Reproduced from [5] with permission from the publisher.

Unfortunately, neither of these factors is likely to be known with adequate accuracy when the clinician evaluates a new patient with VAP in the ICU.

This uncertainty of specific values can be understood using a Monte Carlo simulation. At a casino in Monte Carlo, the results for any given spin of the wheel are unknown, but the distribution of results for a large number of spins is highly predictable. So, a Monte Carlo simulation uses a computer to run through the many thousands of scenarios in which the MIC of the pathogen varies according to the known distribution for likely pathogens. The pharmacokinetics of a drug in a patient also vary within the range expected, based on prior information regarding the measure of central tendency and dispersion for pharmacokinetic parameters values within a population. A dose can then be chosen that will attain the goal of therapy with a probability that is acceptable to the clinician.

The total dose of an antibiotic is not always the most important determinant of success. For $\beta$-lactam antibiotics administered i.v., the period of time over which they are infused is important. When drugs are given as a bolus, regardless of the route, their concentration rapidly reaches a peak and then declines. Once it is $<\mathrm{MIC}$, the drug is no longer effective. If the drug is infused, for example, over a 4 -h period at 8 -h dosing intervals, any infusion rate $\left(\mathrm{mg} \cdot \mathrm{h}^{-1}\right)$ that is able to raise the concentration to $\geqslant$ MIC early in the infusion would then have an effective concentration for $\sim 50 \%$ of the dosing interval. This does not mean, however, that an individual's variable pharmacokinetics are no longer important, because they will affect the infusion rate needed to exceed the MIC. An effective $\% \mathrm{~T}>\mathrm{MIC}$ can be achieved with a lower total dose; reduced cost and toxicity will follow.

Figure 2 shows that the percentage of patients attaining the target associated with maximal cell kill, $40 \% \mathrm{~T}>\mathrm{MIC}$, with an infusion of a 1,000 $\mathrm{mg}$ dose of meropenem would depend on both the MIC and the duration of the infusion [5]. A Monte Carlo simulation estimated the rate of target attainment over a range of pathogen MICs $\left(0.01-100 \mathrm{mg} \cdot \mathrm{L}^{-1}\right)$ for four different infusion lengths, $0.5,1.0,2.0$ and $3.0 \mathrm{~h} q 8 \mathrm{~h}$ (every $8 \mathrm{~h} ; 3$ times daily). The curves show that for higher MICs, the longer the period over which the fixed dose is infused, the more likely it is that the pharmacodynamic target will be achieved. For example, at an MIC of $4 \mathrm{mg} \cdot \mathrm{L}^{-1}$, a 30 -min infusion would reach the $40 \% \mathrm{~T}>$ MIC target in $60 \%$ of the patients, but a 3-h infusion would reach the kill target in $\sim 100 \%$ of patients. For an $8 \mathrm{mg} \cdot \mathrm{L}^{-1}$ MIC pathogen, the $40 \% \mathrm{~T}>\mathrm{MIC}$ target would be reached in $25 \%$ of the patients with a $30-$ min infusion but in $\sim 80 \%$ of the patients with a 3-h infusion. Figure 2 also shows the distribution of the MICs for 8,000 P. aeruginosa isolates. In total, $\geqslant 10 \%$ of the isolates have MICs $\geqslant 4 \mathrm{mg} \cdot \mathrm{L}^{-1}$. At institutions where most pathogens are relatively sensitive (with low

TABLE 2 Meropenem target attainment against Pseudomonas aeruginosa using 1-h versus 3-h infusions at two different doses

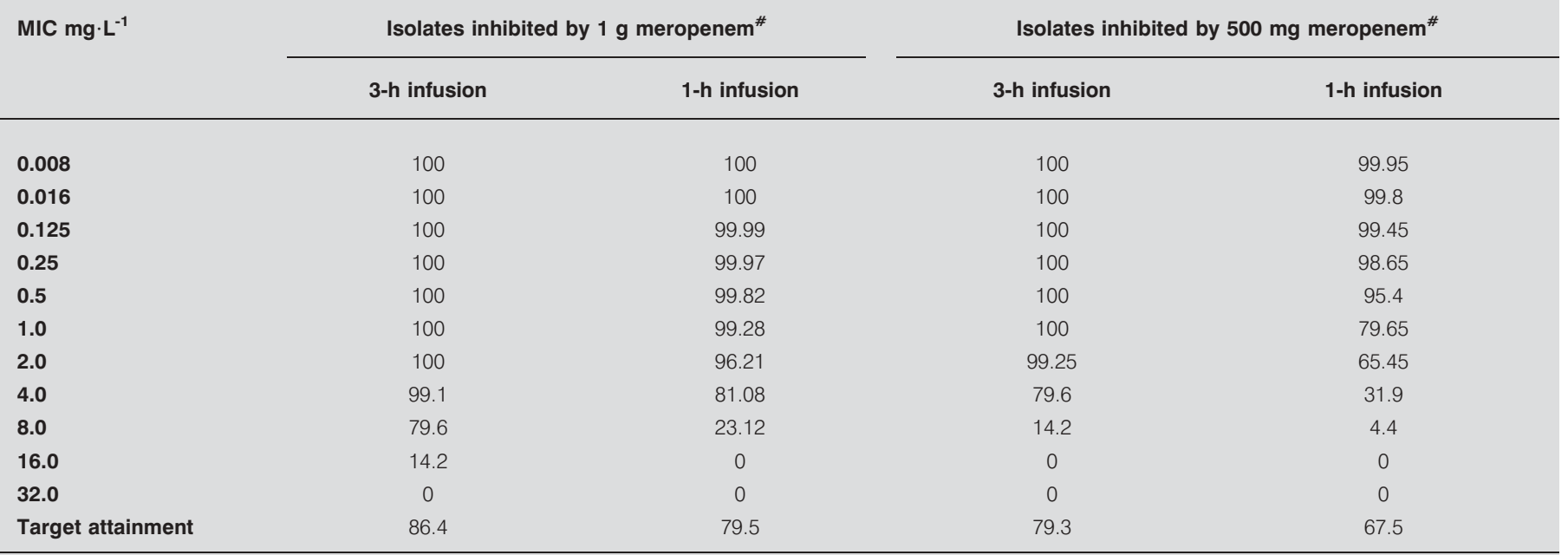

Data are presented as \%. MIC: mean inhibitory concentration. *: every 8 h, 3 times daily. Reproduced from [6] with permission from the publisher 


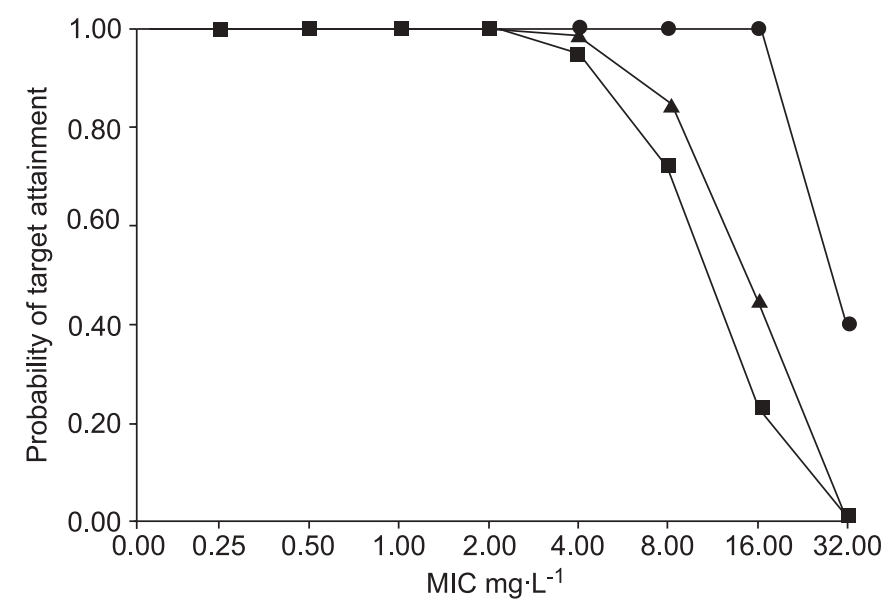

FIGURE 3. Probability of target attainment ( $50 \%$ of the dosing time (T) $>$ mean inhibitory concentration (MIC)) with piperacillin-tazobactam by Monte Carlo simulation. - 30 -min infusion of $3.375 \mathrm{~g}$ every $6 \mathrm{~h} ; \mathbf{\Lambda}: 30$-min infusion of $3.375 \mathrm{~g}$ every $4 \mathrm{~h}$; $: 4$ - $\mathrm{h}$ infusion of $3.375 \mathrm{~g}$ every $8 \mathrm{~h}$. Reproduced from [7] with permission from the publishers.

MICs), the infusion duration may not matter greatly, but for other institutions with a higher proportion of higher MICs, longer infusions will be required to attain $40 \% \mathrm{~T}>\mathrm{MIC}$.

Table 2 presents the target attainment $(40 \% \mathrm{~T}>\mathrm{MIC})$ of meropenem against $P$. aeruginosa comparing a 1-h infusion with an extended 3-h infusion at two different doses (500 $\mathrm{mg}$ or 1,000 mg $q 8$ h) [6]. The analyses were based on MIC distributions from 6,500 strains of $P$. aeruginosa reported as a cause of nosocomial infection in the global Meropenem Yearly Susceptibility Testing Information Collection database for 1997-2002. In highly susceptible $P$. aeruginosa (MIC $<1.0 \mathrm{mg} \cdot \mathrm{L}^{-1}$ ), there is little difference at either dose between the 1-h and the extended 3-h infusion. However, in the more resistant $P$. aeruginosa (MIC $\geqslant 2 \mathrm{mg} \cdot \mathrm{L}^{-1}$ ), the extended infusion was substantially better pharmacodynamically. At MIC $=4 \mathrm{mg} \cdot \mathrm{L}^{-1}$, the $1-\mathrm{h}$ infusion of $1,000 \mathrm{mg}$ attained the target $40 \% \mathrm{~T}>\mathrm{MIC}$ in $81 \%$ of simulated patients, but a 3-h infusion attained this target in $99 \%$ of simulated patients. At a dose of $500 \mathrm{mg}$, target attainment was reached in $80 \%$ of patients with the extended infusion, but in only $32 \%$ with a 1 -h infusion. At MIC $=8 \mathrm{mg} \cdot \mathrm{L}^{-1}$, with the infusion of $1,000 \mathrm{mg}$, target attainment was achieved in 80 versus $23 \%$ of patients with a 1 -h and $3-h$ infusion, respectively; however, with $500 \mathrm{mg}$, it was very low with both infusions. These data illustrate that prolonging infusion substantially improves the chance of microbiological coverage when treating more resistant strains [6].

\section{EXTENDED INFUSIONS IN THE CLINICAL SETTING}

An alternative dosing scheme for piperacillin-tazobactam was designed to optimise the likelihood that $\% \mathrm{~T}>\mathrm{MIC}$ would be $50 \%$ [7]. Instead of $3.375 \mathrm{~g}$ infused for $30 \mathrm{~min} q 4 \mathrm{~h}$ (every $4 \mathrm{~h}$ ) or $q 6 h$ (every $6 \mathrm{~h}$ ), $3.375 \mathrm{~g}$ was administered as an extended (4-h) infusion $q 8 \mathrm{~h}$. The Monte Carlo simulations in figure 3 (using pharmacokinetic data from hospitalised patients) of the probability of achieving this target for various pathogen MICs, found that the 4-h infusion $q 8 \mathrm{~h}$ of piperacillin-tazobactam had a $>90 \%$ probability of achieving the $50 \% \mathrm{~T}>$ MIC target, at
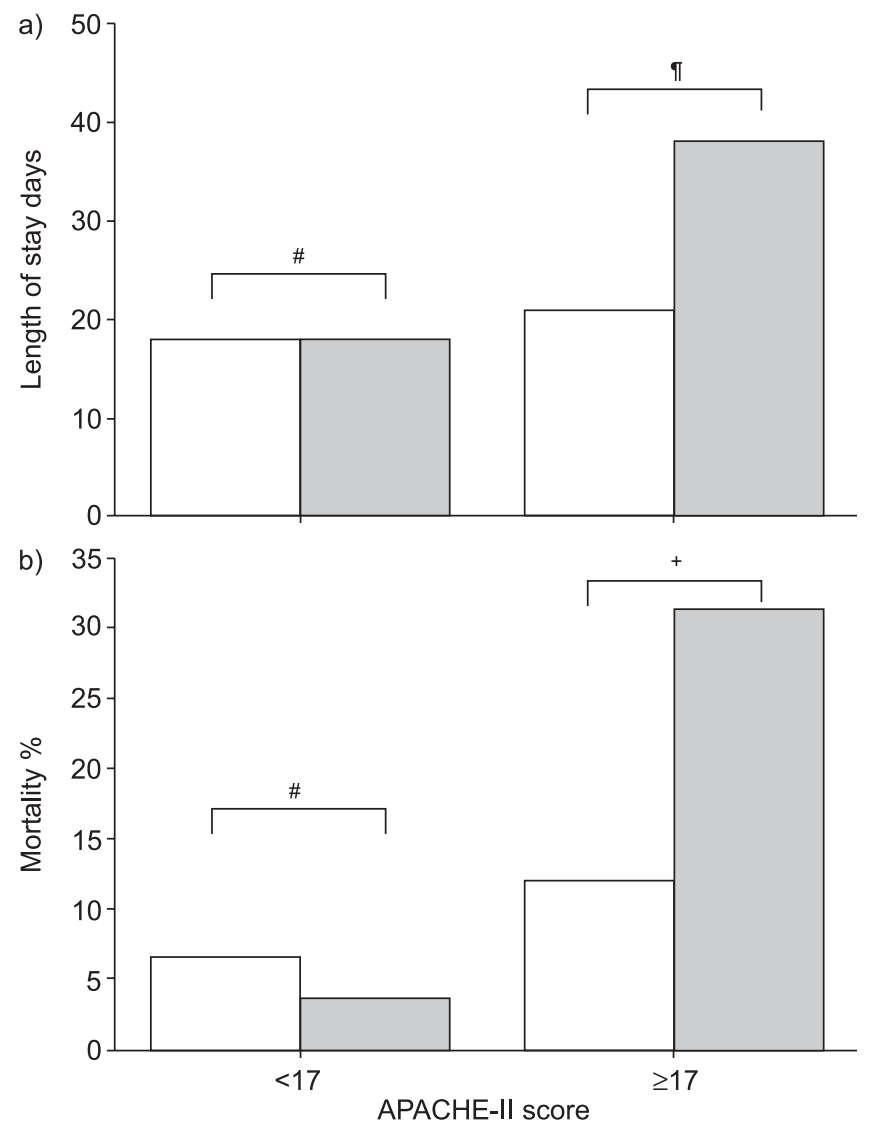

FIGURE 4. a) Length of hospital stay for patients who lived $\geqslant 14$ days after the first Pseudomonas aeruginosa culture was collected. b) Comparison of 14-day mortality after the first $P$. aeruginosa culture was collected. APACHE: Acute Physiological and Chronic Health Evaluation. $n=61$ and 41 for the extended infusion ( $\square$ ) in patients with APACHE scores $<17$ and $\geqslant 17$, respectively. $n=54$ and 38 for the intermittent infusion $(\square)$ in patients with APACHE scores $<17$ and $\geqslant 17$, respectively. ${ }^{\#:}$ nonsignificant; ${ }^{9}: p=0.02 ;^{+}: p=0.04$. Reproduced from [7] with permission from the publisher.

MICs $\leqslant 16 \mathrm{mg} \cdot \mathrm{L}^{-1}$. With a 0.5 -h infusion $q 4 h$, the probability of reaching the target was $<80 \%$ when the MIC was $\geqslant 16 \mathrm{mg} \cdot \mathrm{L}^{-1}$. With a 0.5 -h infusion $q 6 h$, the probability of reaching the target was $<80 \%$ when the MIC was $\geqslant 8 \mathrm{mg} \cdot \mathrm{L}^{-1}$.

In 2002, a protocol was implemented specifying that all orders for a 30-min infusion were to be automatically converted to the extended-infusion dose and schedule. To evaluate the effects of this change, clinical outcomes of patients with $P$. aeruginosa infections during the first $2 \mathrm{yrs}$ following the switch were compared with those from the preceding 2 yrs. Patients in each treatment group were divided further into subjects with Acute Physiological and Chronic Health Evaluation-II (APACHE-II) scores $<17$ and subjects with APACHE-II scores $\geqslant 17$ (total of four groups). Figure 4 illustrates that in patients with APACHE-II $<17$, neither length of stay (18 days for both) nor mortality ( 3.7 versus $6.6 \%$ ) were different between treatments. For patients with APACHE-II scores $\geqslant 17$, however, the extended infusion reduced both the length of stay (from 38 to 21 days; $p=0.02$ ) and mortality (from 31.6 to $12.2 \%$; 
$p=0.04$ ). Only the more ill patients recovered significantly more quickly with the extended infusion.

\section{DORIPENEM, AN INVESTIGATIONAL CARBAPENEM}

The principles of extended infusions are broadly applicable for $\beta$-lactam therapy. The relationship between pharmacodynamic/pharmacokinetic parameters and experimental in vivo efficacy of doripenem, an investigational carbapenem, was measured in the neutropenic murine thigh infection model [8]. Data from Staphylococcus aureus, Streptococcus pneumoniae and several Gram-negative bacilli (including Klebsiella pneumoniae) demonstrated a significant correlation between the change in CFU thigh $^{-1}$ and the $\% \mathrm{~T}>\mathrm{MIC}$, similar to data from other $\beta$-lactams and carbapenems. There was no significant correlation between the change in CFU.thigh ${ }^{-1}$ in either the area under the curve/MIC ratio or the peak concentration/MIC ratio. The target $2-\log$ kill $\% \mathrm{~T}>\mathrm{MIC}$ mean was $27.3 \%$ for $S$. pneumoniae, $35.4 \%$ for $S$. aureus and $43.7 \%$ for Gram-negative bacilli [8].

In multicentre phase III studies of hospital-acquired infections, in which less susceptible pathogens such as P. aeruginosa are common, doripenem will be compared with other carbapenems, which are less stable than doripenem following reconstitution [9-11]. The clinical utility of prolonged infusion will be evaluated and may provide significant insight into the utility of such regimens for $\beta$-lactam agents.

\section{CONCLUSIONS}

Rational dose selection is improved by the use of pharmacodynamic data and, in particular, by the use of Monte Carlo simulations to determine the probability that a given antibiotic dose and dosing regimen will achieve the targeted kill. The appropriate pharmacodynamic target for $\beta$-lactams is the percentage of dosage time spent above the minimum inhibitory concentration, and carbapenems achieve microbiological goals at a lower percentage of time above the minimum inhibitory concentration than do other members of the $\beta$-lactam class. Extended infusion of i.v. $\beta$-lactams improves attainment of pharmacodynamic targets at a given dose, which may be expected to increase efficacy. Extended infusion may give better results (lower mortality) in severely ill patients, while still using lower doses. A lower dose of antibiotic decreases both the cost and expected toxicity. Thus, extended infusion should be considered whenever an i.v. $\beta$-lactam is used for treatment of infections caused by difficult pathogens; drugs with high stability are particularly suitable for extended infusion.

\section{REFERENCES}

1 Patel PJ, Leeper KV Jr, McGowan JE Jr. Epidemiology and microbiology of hospital-acquired pneumonia. Semin Respir Crit Care Med 2002; 23: 415-425.

2 Cavalcanti M, Valencia M, Torres A. Respiratory nosocomial infections in the medical intensive care unit. Microbes Infect 2005; 7: 292-301.

3 Safdar N, Dezfulian C, Collard HR, Saint S. Clinical and economic consequences of ventilator-associated pneumonia: a systematic review. Crit Care Med 2005; 33: 2184-2193.

4 American Thoracic Society; Infectious Diseases Society of America. Guidelines for the management of adults with hospital-acquired, ventilator-associated, and healthcareassociated pneumonia. Am J Respir Crit Care Med 2005; 171: 388-416.

5 Drusano GL. Prevention of resistance: a goal for dose selection for antimicrobial agents. Clin Infect Dis 2003; 36: Suppl. 1, S42-S50.

6 Lomaestro BM, Drusano GL. Pharmacodynamic evaluation of extending the administration time of meropenem using a Monte Carlo simulation. Antimicrob Agents Chemother 2005; 49: 461-463.

7 Lodise TP Jr, Lomaestro B, Drusano GL. Piperacillintazobactam for Pseudomonas aeruginosa infection: clinical implications of an extended-infusion dosing strategy. Clin Infect Dis 2007; 44: 357-363.

8 Andes DR, Kiem S, Craig W. In-vivo pharmacodynamic activity of a new carbapenem, doripenem (DOR), against multiple bacteria in a murine thigh infection model. 43rd Interscience Conference on Antimicrobial Agents and Chemotherapy (ICAAC). Chicago, American Society of Medicine, 2003.

9 Primaxin ${ }^{\circledR}$ I.M. (Imipenem and cilastatin for injectable suspension). Whitehouse Station, USA, Merck and Co., Inc., 2005.

10 Merrem $^{\mathrm{TM}}$ Package Insert. Wilmington, USA, AstraZeneca, 2005.

11 Peninsula, Press Release at http://sec.edgar-online.com/ 2004/02/18/0000950134-04-002233/Section11.asp. Date last accessed: October 11, 2006. 